\title{
High expression levels of unc-51-like kinase 1 as a predictor of poor prognosis in colorectal cancer
}

\author{
YIFENG ZOU*, ZEXIAN CHEN*, XIAOWEN HE, XIAOSHENG HE, XIANRUI WU, \\ YUFENG CHEN, XIAOJIAN WU, JIANPING WANG and PING LAN \\ Department of Colorectal and Anal Surgery, The Sixth Affiliated Hospital, Sun \\ Yat-sen University, Guangzhou, Guangdong 510655, P.R. China
}

Received October 7, 2014; Accepted June 3, 2015

DOI: $10.3892 / \mathrm{ol} .2015 .3417$

\begin{abstract}
Colorectal cancer (CRC) is one of the most common and lethal cancers worldwide. Unc-51-like kinase 1 (ULK1) plays an important role in autophagy, which is widely involved in human CRC. The aim of the present study was to investigate the clinical and prognostic significance of the expression of ULK1 in human CRC. Expression of ULK1 in 339 CRC specimens (tumor-node-metastasis stages I-IV) was assessed by immunohistochemistry. The optimal cutpoint of the expression of ULK1 was assessed by the X-tile program, and the patients were divided into 2 groups of high or low expression levels of ULK1, accordingly. Correlation analysis between the expression of ULK1 and the clinicopathological variables in CRC demonstrated that the expression of ULK1 was significantly associated with gender and tumor differentiation. Univariate Cox regression analysis indicated that high expression levels of ULK1 were a risk factor for overall and disease-free survival. Therefore, the high expression levels of ULK1 may be a useful independent biomarker for predicting a poor prognosis in patients with CRC.
\end{abstract}

\section{Introduction}

Colorectal cancer (CRC) is the third and second most common type of cancer in males and females, respectively (1). In 2008, $>1.2$ million cases of CRC were diagnosed, and 608,700 associated mortalities were recorded (1). Patients with CRC have different prognoses depending on the tumor stage, which is commonly classified by the American Joint Committee on Cancer (AJCC) tumor-node-metastasis (TNM) staging system (2). For example, patients with distant metastasis have a poor 5-year survival rate (12\%), while patients with localized

Correspondence to: Mr. Ping Lan, Department of Colorectal and Anal Surgery, The Sixth Affiliated Hospital, Sun Yat-sen University, 26 Yuancun Erheng Road, Guangzhou, Guangdong 510655,P.R. China E-mail: sumslp@163.com

*Contributed equally

Key words: ULK1, prognosis, colorectal cancer disease have good prognoses (>90\%) (3). However, the AJCC staging system is mainly concerned with the depth of cancer invasion, the involvement of the lymph nodes and the status of the metastasis, but not with the specific biological properties of CRC (4). Thus far, few biological markers have been validated as diagnostic criteria. However, certain molecules involved in the pathogenesis of CRC may lead to more accurate diagnoses and improved efficacy of comprehensive therapies (5).

Autophagy, also known as cellular digestion, is a highly conserved cellular catabolic pathway involved in the degradation and recycling of superfluous or damaged proteins and organelles via double-membrane vesicles termed autophagosomes. This process enables cells, organs and entire organisms to endure various stress conditions, including limited availability of nutrients, reduced energy supply or low levels of oxygen $(6,7)$. Numerous autophagy-associated genes (ATG) have been identified in yeast. Unc-51-like kinase 1 (ULK1) is a core mammalian homologue of a yeast ATG. In mammalian cells, ULK1 forms a stable complex to sense nutrient signals for autophagy activation (8). Under conditions of glucose starvation, the activated AMP-activated protein kinase (AMPK) regulates the ULK1 complex by direct interaction as follows: AMPK phosphorylates and activates ULK1, which leads to autophagy induction $(9,10)$. When glucose levels are sufficient, high levels of mammalian target of rapamycin complex 1 prevent ULK1 activation $(11,12)$. The role of autophagy in cancer is complex, and varies depending on the type of tumor. For example, high expression levels of ULK1 have been demonstrated to be a biomarker of poor prognosis in patients with esophageal squamous cell carcinoma (13); and in operable breast cancer, low expression levels of ULK1 are associated with tumor progression and an adverse prognosis (14). Thus far, the association between the expression of ULK1 and the pathogenesis of CRC remains unclear.

To the best of our knowledge, the characterization of the expression of ULK1 in human CRC tissues, and its association with the clinicopathological variables of CRC are reported for the first time in the present study.

\section{Materials and methods}

Patients and follow-up. The current study was approved by the Institute Research Medical Ethics Committee of Sun Yat-sen University (Guangzhou, China). A total of 339 patients 
with CRC, who underwent initial surgical resection in the Department of Gastrointestinal Surgery of the First Affiliated Hospital of Sun Yat-sen University between January 2003 and December 2005, were enrolled in the present study. Written informed consent was obtained from each patient. Patients that had received preoperative chemo- or radiotherapy were excluded. The cancer tissues were surgically resected and histopathologically confirmed by hematoxylin and eosin (H\&E; Beyotime Institute of Biotechnology, Haimen, China) staining.

During the follow-up, the patients were evaluated at the hospital or contacted by telephone or letter every 3 months in the first year, every 6 months in the second year and annually thereafter. The following data were collected from the patients for further investigation: General information, preoperative information, details of the surgery, pathology reports, TNM stage, and results of the follow-ups. The tumor stage was defined according to the AJCC TNM stage system of CRC ( $7^{\text {th }}$ edition) (2). For follow-up purposes, the primary end point was the overall survival (OS), defined as the time from surgery to mortality due to any cause; and the secondary end point was the disease-free survival (DFS), defined as the time from surgery to the first event of recurrence, metastasis or mortality.

Tissue microarray (TMA) construction and immunohistochemical (IHC) staining. TMA was constructed using paraffin-embedded tissue blocks of cancer tissues following screening of the H\&E-stained slides for optimal position: For each specimen, 2 cores of 1-mm diameter were excised from the representative areas and deposited on a recipient paraffin block using a Minicore tissue array (ALPHELYS, Plaisir, France). The blocks were subsequently cut into 5- $\mu \mathrm{m}$ sections on silanized glass slides (Beyotime Institute of Biotechnology) for IHC staining.

IHC staining was performed with an Envision system, according to the manufacturer's instructions (Dako, Glostrup, Denmark): The slides were deparaffinized in dimethylbenzene and rehydrated with graded alcohol $(100 \%, 95 \%, 75 \%)$ prior to antigen retrieval with sodium citrate (Sangon Biotech Co., Ltd., Shanghai, China) and blocking of the endogenous peroxidase activity with $0.3 \%$ hydrogen peroxide (Sangon Biotech Co., Ltd.). The slides were then incubated overnight at $4^{\circ} \mathrm{C}$ with a polyclonal rabbit ULK1 antibody (1:2,000; \#ab65056; Abcam, Cambridge, UK), followed by a 30-min incubation period at room temperature with a polyclonal goat anti-rabbit secondary antibody (\#A0208; Beyotime Institute of Biotechnology). Next, the slides were rinsed with phosphate-buffered saline, incubated with 3,3'-diaminobenzidine for $1 \mathrm{~min}$, counterstained with hematoxylin, dehydrated using graded alcohol $(90 \%, 95 \%, 100 \%)$ and mounted. A negative control was obtained by replacing the primary antibody with a normal murine IgG (Beyotime Institute of Biotechnology), whereas positively staining slides were used as positive controls.

Evaluation of IHC analysis and selection of cutpoint value. Following IHC staining, digital images of each spot in the slides were captured at a magnification of x 200 using a DMI4000B inverted research microscope (Leica Microsystems GmbH, Wetzlar, Germany). In order to measure the expression levels of ULK1, the tumor area was selected and then analyzed with the TMAJ software (TMA Core Facility,
Johns Hopkins University, Baltimore, MD, USA), which discriminated the immunostained area by hue, saturation and brightness color range. As a result, a numerical value known as the ULK1 expression index was obtained, which corresponds to the density of the expression of ULK1 multiplied by the intensity of the staining. The mean ULK1 expression index for each of the duplicate cores was used for further statistical analysis.

In order to optimize the cutpoint of the ULK1 expression index according to the clinicopathological data of the patients, an open source software termed X-tile program, version 3.6.1 (School of Medicine, Yale University, New Haven, CT, USA) was used $(15,16)$. The X-tile program divided the cohorts randomly into a matched training and validation set. Statistical significance was assessed by the log-rank analysis method, using the cutpoint derived from a training set to parse a separate validation set. The X-tile plots determined an optimal cutpoint value, and the correction for the minimum $\mathrm{P}$ statistics was calculated using the Miller-Siegmund P-value correction (17).

Statistical analysis. All the statistical analyses were performed with SPSS software, version 16 (SPSS, Inc., Chicago, IL, USA). The correlation between the expression levels of ULK1 and the clinicopathological features of CRC was analyzed using the $\chi^{2}$ test. The Kaplan-Meier method was used to estimate the OS and DFS. Furthermore, a multivariate Cox proportional hazard regression model was constructed using the enter method with an entry criterion of $\mathrm{P}<0.05$ and a removal criterion of $\mathrm{P}>0.10$. $\mathrm{P}<0.05$ (2-sided) was considered to indicate a statistically significant difference.

\section{Results}

Characteristics of the eligible patients. As presented in Table I, a total of 339 patients with CRC (184 males and 155 females; mean age, 59.1 years) were enrolled for IHC analysis in the present study. Based on the AJCC TNM staging system, there were 51, 142, 118 and 28 patients with stages I, II, III and IV CRC, respectively. The locations of the tumors were the colon and rectum in 167 and 172 patients, respectively. Following a median follow-up period of 60 months, 90 patients deceased. Table I indicates the characteristics of the eligible patients.

Correlation between ULK1 and clinicopathological variables. Representative images of tissues expressing ULK1 are presented in Fig. 1A. ULK1 was detected in the cytoplasm of cancer cells. To assess the homogeneity and reliability of the IHC results, the data derived from the TMAJ software were further analyzed by the Pearson's product-moment correlation coefficient, which demonstrated a significant correlation $(r=0.738 ; \mathrm{P}<0.001)$ between the expression levels of ULK1 and the clinicopathological variables of the patients with CRC (Fig. 1B). According to the analysis performed by the $\mathrm{X}$-tile program, high and low expression levels of ULK1 were observed in 271 and 68 patients, respectively.

High expression levels of ULK1 were significantly correlated with gender $(\mathrm{P}=0.031)$, poor tumor differentiation $(\mathrm{P}=0.029)$, high expression levels of preoperative carcinoembryonic antigen (CEA) $(\mathrm{P}=0.067)$ and advanced TNM stage $(\mathrm{P}=0.085)$. Table II 
Table I. Characteristics of 339 patients with colorectal cancer (mean age, $59.10 \pm 0.74$ years).

\begin{tabular}{lc} 
Variables & Frequency, n $(\%)$ \\
\hline Gender & \\
Male & $184(54.3 \%)$ \\
Female & $155(45.7 \%)$ \\
TNM stage & \\
I & $51(15.0 \%)$ \\
II & $142(41.9 \%)$ \\
III & $118(34.8 \%)$ \\
IV & $28(8.3 \%)$ \\
Tumor location & \\
Colon & $167(49.3 \%)$ \\
Rectum & $172(50.7 \%)$ \\
ULK1 expression levels & \\
Low & $68(20.1 \%)$ \\
High & $271(79.9 \%)$
\end{tabular}

TNM, tumor node metastasis; ULK1, unc-51-like kinase 1.

indicates the correlation between the expression levels of ULK1 and the clinicopathological characteristics of patients with CRC.

Association between ULK1 and survival status. The Kaplan-Meier analysis and the univariate Cox proportional hazard regression model were used to investigate the impact of the expression levels of ULK1 on the OS (Table III) and DFS (Table IV) of patients with CRC. The results demonstrated that high expression levels of ULK1 were significantly correlated with adverse OS (log-rank test, $\mathrm{P}=0.035$ ) and $\mathrm{DFS}$ (log-rank test, $\mathrm{P}=0.022)$. Other clinicopathological variables, including preoperative levels of CEA and carbohydrate antigen 19-9 (CA199) $(\mathrm{P}<0.001$ and $\mathrm{P}=0.004$, respectively), TNM stage $(\mathrm{P}<0.001)$, differentiation $(\mathrm{P}=0.001)$ and postoperative recurrence or metastasis $(\mathrm{P}<0.001)$ were also observed to be significant indicators of the OS of patients with CRC, whereas preoperative levels of CEA and CA199 $(\mathrm{P}<0.001$ and $\mathrm{P}=0.002$, respectively), TNM stage $(\mathrm{P}<0.001)$ and differentiation $(\mathrm{P}<0.001)$ were observed to significantly affect the DFS of patients with CRC.

Additionally, multivariate analysis was performed using the Cox proportional hazards model for all of the variables observed to be significant in the univariate analysis. The results indicated that the preoperative levels of CEA and the TNM stage remained significant independent adverse prognostic factors for OS ( $\mathrm{P}=0.013$ and $<0.001$, respectively) and DFS $(\mathrm{P}=0.005$ and $<0.001$, respectively), and that the postoperative recurrence or metastasis was an independent prognostic factor for OS $(\mathrm{P}=0.001)$. However, the expression levels of ULK1 were not observed to be an independent prognostic factor for OS or DFS ( $\mathrm{P}=0.173$ and 0.118 , respectively). The association between the variables and the survival status is presented in Tables III and IV. The survival curves obtained according to the expression levels of ULK1 in patients with CRC are represented in Fig. 2.
A
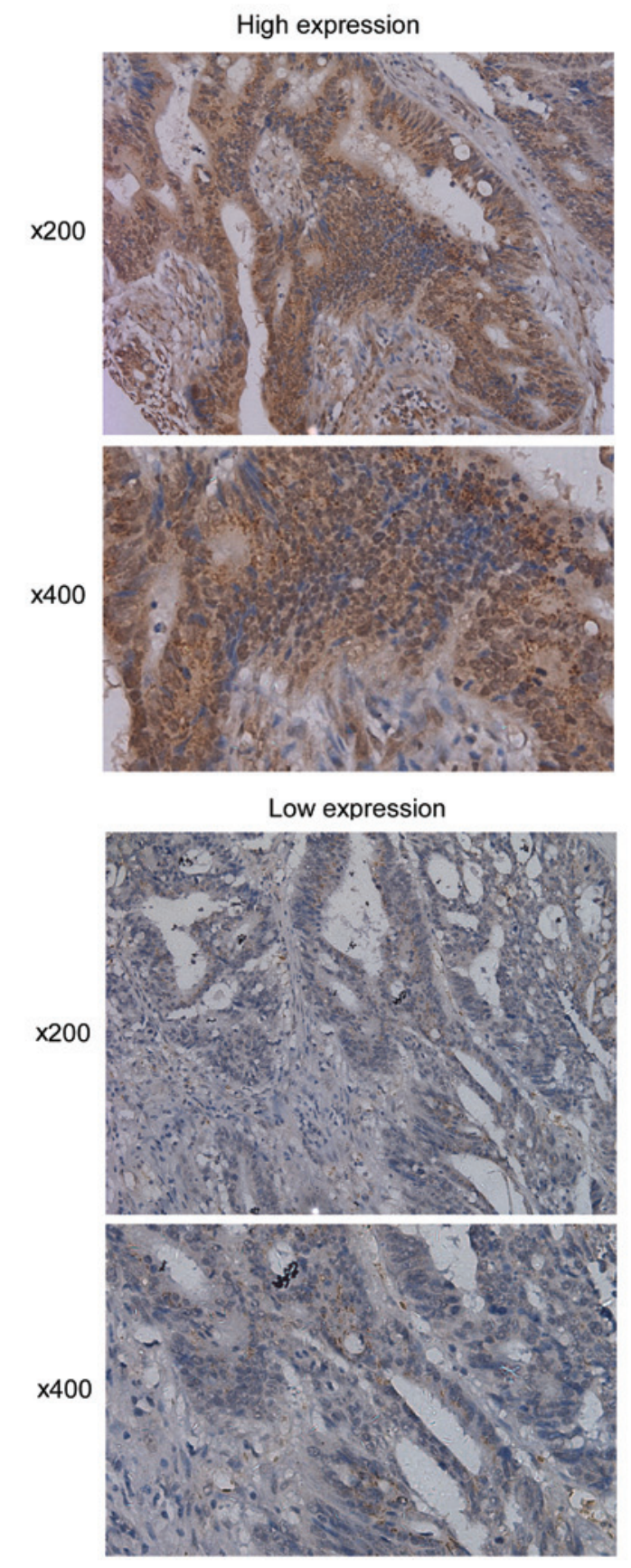

B

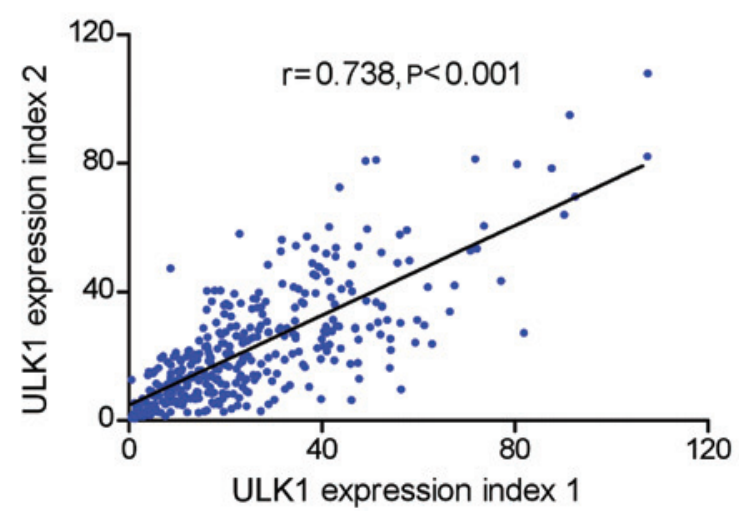

Figure 1. Immunohistochemical staining of the expression of ULK1 in human colorectal cancer. (A) ULK1 expression was localized within the cytoplasm of cancer cells, as demonstrated by the representative images of tissues with high (left) and low (right) expression levels of ULK1, [magnification, x200 (top) and x400 (bottom)] (B) Homogeneity of the expression of ULK1 ( $\mathrm{r}=0.738 ; \mathrm{P}<0.001)$. ULK1, unc-51-like kinase 1. 
Table II. Correlation between the expression levels of ULK1 and the clinicopathological characteristics of patients with colorectal cancer.

\begin{tabular}{|c|c|c|c|c|}
\hline \multirow[b]{2}{*}{ Variable } & \multirow[b]{2}{*}{ Cases } & \multicolumn{2}{|c|}{ ULK1 expression levels } & \multirow[b]{2}{*}{ P-value } \\
\hline & & Low & High & \\
\hline \multicolumn{5}{|l|}{ Gender } \\
\hline Female/male & $155 / 184$ & $39 / 29$ & $116 / 155$ & 0.031 \\
\hline \multicolumn{5}{|l|}{ Age, years } \\
\hline$<60 / \geq 60$ & $164 / 175$ & $30 / 38$ & $134 / 137$ & 0.432 \\
\hline \multicolumn{5}{|l|}{$\mathrm{CEA}, \mathrm{ng} / \mathrm{ml}$} \\
\hline$<5 / \geq 5$ & $233 / 106$ & $53 / 15$ & $180 / 91$ & 0.067 \\
\hline \multicolumn{5}{|l|}{ CA199, U/ml } \\
\hline$<37.5 / \geq 37.5$ & $265 / 74$ & $57 / 11$ & $208 / 63$ & 0.207 \\
\hline \multicolumn{5}{|l|}{ pT } \\
\hline $\mathrm{T} 1+\mathrm{T} 2 / \mathrm{T} 3+\mathrm{T} 4$ & $62 / 277$ & $17 / 51$ & $45 / 226$ & 0.109 \\
\hline \multicolumn{5}{|l|}{$\mathrm{pN}$} \\
\hline $\mathrm{N} 0 / \mathrm{N} 1+\mathrm{N} 2$ & $206 / 133$ & $47 / 21$ & $159 / 112$ & 0.115 \\
\hline \multicolumn{5}{|l|}{ pM } \\
\hline M0/M1 & $311 / 28$ & $66 / 2$ & $245 / 26$ & 0.125 \\
\hline \multicolumn{5}{|l|}{ TNM stage } \\
\hline $\mathrm{I}+\mathrm{II} / \mathrm{III}+\mathrm{IV}$ & $193 / 146$ & $45 / 23$ & $148 / 123$ & 0.085 \\
\hline \multicolumn{5}{|l|}{ Tumor differentiation } \\
\hline Well+moderately/poorly & $301 / 38$ & $66 / 2$ & $235 / 36$ & 0.029 \\
\hline
\end{tabular}

ULK1, unc-51-like kinase 1; CEA, carcinoembryonic antigen; CA199, carbohydrate antigen 19-9; TNM, tumor node metastasis stage; pTNM, pathological TNM.

Table III. Univariate and multivariate analyses of variables related to overall survival in patients with colorectal cancer.

\begin{tabular}{|c|c|c|c|c|c|c|}
\hline \multirow[b]{2}{*}{ Variable } & \multicolumn{3}{|c|}{ Univariate analysis } & \multicolumn{3}{|c|}{ Multivariate analysis } \\
\hline & $\mathrm{HR}$ & $95 \% \mathrm{CI}$ & P-value & $\mathrm{HR}$ & $95 \% \mathrm{CI}$ & P-value \\
\hline Gender & & & & & & \\
\hline Male/female & 1.083 & $0.719-1.631$ & 0.704 & & & \\
\hline $\begin{array}{l}\text { Age, years } \\
\geq 60 /<60\end{array}$ & 1.443 & $0.953-2.186$ & 0.084 & & & \\
\hline $\begin{array}{l}\text { CEA, ng/ml } \\
\geq 5 /<5\end{array}$ & 2.206 & $1.452-3.351$ & $<0.001$ & 1.769 & $1.126-2.777$ & 0.013 \\
\hline $\begin{array}{l}\text { CA199, U/ml } \\
\geq 37.5 /<37.5\end{array}$ & 1.932 & $1.236-3.020$ & 0.004 & 1.274 & $0.787-2.063$ & 0.324 \\
\hline $\begin{array}{l}\text { TNM stage } \\
\text { III+IV/I+II }\end{array}$ & 2.980 & $1.953-4.546$ & $<0.001$ & 2.294 & $1.465-3.590$ & $<0.001$ \\
\hline $\begin{array}{l}\text { Differentiation } \\
\text { Poorly/well+moderately }\end{array}$ & 2.476 & $1.478-4.151$ & 0.001 & 1.597 & $0.929-2.743$ & 0.090 \\
\hline $\begin{array}{l}\text { Postoperative recurrence o } \\
\text { Present/absent }\end{array}$ & 3.483 & $2.209-5.492$ & $<0.001$ & 2.274 & $1.407-3.676$ & 0.001 \\
\hline $\begin{array}{l}\text { ULK1 expression levels } \\
\text { High/low }\end{array}$ & 1.923 & $1.048-3.526$ & 0.035 & 1.535 & $0.828-2.846$ & 0.173 \\
\hline
\end{tabular}

CEA, carcinoembryonic antigen; CA199, carbohydrate antigen 19-9; HR, hazard ratio; CI, confidence interval; TNM, tumor node metastasis; ULK1, unc-51-like kinase 1. 
Table IV. Univariate and multivariate analyses of variables related to disease-free survival in patients with colorectal cancer.

\begin{tabular}{|c|c|c|c|c|c|c|}
\hline \multirow[b]{2}{*}{ Variable } & \multicolumn{3}{|c|}{ Univariate analysis } & \multicolumn{3}{|c|}{ Multivariate analysis } \\
\hline & HR & $95 \% \mathrm{CI}$ & P-value & HR & $95 \%$ CI & P-value \\
\hline \multicolumn{7}{|l|}{ Gender } \\
\hline Female/male & 1.084 & $0.743-1.583$ & 0.675 & & & \\
\hline Age, years & & & & & & \\
\hline$<60 / \geq 60$ & 1.248 & $0.854-1.824$ & 0.251 & & & \\
\hline $\begin{array}{l}\text { CEA, ng/ml } \\
<5 / \geq 5\end{array}$ & 2.126 & $1.440-3.137$ & $<0.001$ & 1.836 & $1.202-2.805$ & 0.005 \\
\hline $\begin{array}{l}\mathrm{CA} 199, \mathrm{U} / \mathrm{ml} \\
<37.5 / \geq 37.5\end{array}$ & 1.939 & $1.278-2.943$ & 0.002 & 1.345 & $0.856-2.115$ & 0.198 \\
\hline $\begin{array}{l}\text { TNM stage } \\
\mathrm{I}+\mathrm{II} / \mathrm{III}+\mathrm{IV}\end{array}$ & 2.923 & $1.982-4.310$ & $<0.001$ & 2.495 & $1.677-3.714$ & $<0.001$ \\
\hline $\begin{array}{l}\text { Differentiation } \\
\text { Well+moderately/poorly }\end{array}$ & 2.649 & $1.657-4.236$ & $<0.001$ & 1.914 & $1.173-3.125$ & 0.009 \\
\hline $\begin{array}{l}\text { ULK1 expression levels } \\
\text { Low/high }\end{array}$ & 1.930 & $1.101-3.384$ & 0.022 & 1.576 & $0.891-2.786$ & 0.118 \\
\hline
\end{tabular}

CEA, carcinoembryonic antigen; CA199, carbohydrate antigen 19-9; HR, hazard ratio; CI, confidence interval; TNM, tumor mode metastasis; ULK1, unc-51-like kinase 1 .
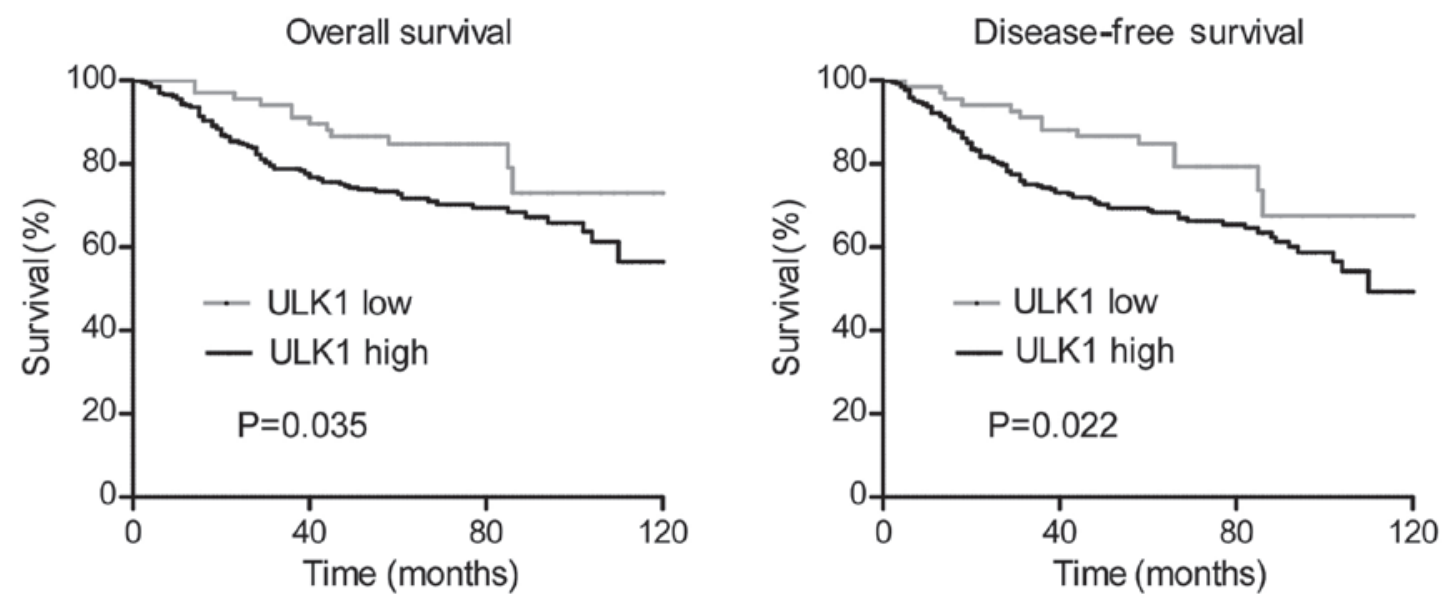

Figure 2. Survival analysis of patients with colorectal cancer who present high expression levels of ULK1 vs. those with low expression levels of ULK1. The overall (left) and disease-free survival (right) were observed to be significantly lower in patients with high expression levels of ULK1 (P=0.035 and 0.022 , respectively). The P-values were calculated using the univariate analyses (log-rank test). ULK1, unc-51-like kinase 1.

\section{Discussion}

CRC is a common malignancy worldwide (1). Despite the improvements in diagnostic techniques and therapeutic methods, CRC remains a serious challenge (18). Currently, the treatment and prognosis of patients with CRC depends on the AJCC TNM stage classification system, according to the results of pathological analysis (2). However, this staging system is not sufficiently reliable. Different studies have demonstrated that the OS of patients with CRC of stage IIb was poorer than those of stage IIIa (19-21). As an alternative to the AJCC TNM staging system, an increasing number of molecular biomarkers have been defined as useful prognostic and predictive factors in CRC (22), including KARS and BRAF, which are commonly used in clinical practice $(23,24)$.

Autophagy is a cellular dynamic process involved in the regulation of carcinogenesis and the response to anticancer therapy $(25,26)$. ULK1, as a core ATG, is involved in the initiation of autophagy (27). The role of ULK1 in different tumors varies with the type of cancer $(13,14)$. To the best of our knowledge, the present study reports for the first time that the expression levels of ULK1 increase with the magnitude of cancer progression in tissue specimens derived from patients with CRC, according to the results of IHC staining.

In the current study, high expression levels of ULK1 were observed to be significantly associated with female gender 
and poor tumor differentiation, and were slightly associated with high expression levels of preoperative CEA and advanced TNM stage. In the survival analysis, high expression levels of ULK1 were significantly correlated with adverse OS and DFS in the univariate analysis, but not in the multivariate analysis. These results suggest that the expression levels of ULK1 are of clinical value to assess the prognosis and survival of patients with CRC.

The majority of cancer cells grow preferentially in the presence of abundant nutrients and oxygen, which facilitate a high proliferation rate (13). However, CRC is an example of a common solid tumor with a limited blood supply, and the resulting metabolic stress becomes more severe as the cancer develops (28). Since the autophagy process maintains cellular homeostasis and degrades toxic cytoplasmic constituents, it also enables malignant cells to endure various stress conditions, including limited availability of nutrients, reduced energy supply or low levels of oxygen $(6,7)$. Consistently, the cancer cells may express high levels of ULK1 in order to activate the initiation of autophagy and survive. This may explain why high expression levels of ULK1 in patients with CRC indicate a poor prognosis. However, further studies are required to clarify the exact mechanism of action.

In conclusion, the current study demonstrates that high expression levels of ULK1 may be a useful independent biomarker for predicting the poor prognosis of patients with CRC.

\section{Acknowledgements}

The current work was supported by the National Natural Science Foundation of China (No. 81300367) and the Natural Science Foundation of Guangdong Province (No. S2013010014186).

\section{References}

1. Jemal A, Bray F, Center MM, Ferlay J, Ward E and Forman D: Global cancer statistics. CA Cancer J Clin 61: 69-90, 2011.

2. Edge SB and Compton CC: The American Joint Committee on Cancer: The 7th edition of the AJCC cancer staging manual and the future of TNM. Ann Surg Oncol 17: 1471-1474, 2010.

3. Siegel R, Ward E, Brawley O and Jemal A: Cancer statistics, 2011: The impact of eliminating socioeconomic and racial disparities on premature cancer deaths. CA Cancer J Clin 61: 212-236, 2011

4. Nagtegaal ID and Quirke P: Revised staging: Is it really better, or do we not know? J Clin Oncol 28: e397-e400, 2010

5. Graziano F and Cascinu S: Prognostic molecular markers for planning adjuvant chemotherapy trials in Dukes' B colorectal cancer patients: How much evidence is enough? Ann Oncol 14: 1026-1038, 2003.

6. Zhao M and Klionsky DJ: AMPK-dependent phosphorylation of ULK1 induces autophagy. Cell Metab 13: 119-120, 2011.

7. Mizushima N, Levine B, Cuervo AM and Klionsky DJ: Autophagy fights disease through cellular self-digestion. Nature 451: 1069-1075, 2008.

8. Wong PM, Puente C, Ganley IG and Jiang X: The ULK1 complex: Sensing nutrient signals for autophagy activation. Autophagy 9: $124-137,2013$
9. Kim J, Kundu M, Viollet B and Guan KL: AMPK and mTOR regulate autophagy through direct phosphorylation of Ulk1. Nat Cell Biol 13: 132-141, 2011.

10. Lee JW, Park S, Takahashi Y and Wang HG: The association of AMPK with ULK1 regulates autophagy. PLoS One 5: e15394, 2010.

11. Hosokawa N, Hara T, Kaizuka T, Kishi C, Takamura A, Miura Y, Iemura S, Natsume T, Takehana K, Yamada N, et al: Nutrient-dependent mTORC1 association with the ULK1-Atg13-FIP200 complex required for autophagy. Mol Biol Cell 20: 1981-1991, 2009.

12. Jung CH, Jun CB, Ro SH, Kim YM, Otto NM, Cao J, Kundu M and Kim DH: ULK-Atg13-FIP200 complexes mediate mTOR signaling to the autophagy machinery. Mol Biol Cell 20: 1992-2003, 2009.

13. Jiang S, Li Y, Zhu YH, Wu XQ, Tang J, Li Z, Feng GK, Deng R, Li DD, Luo RZ, et al: Intensive expression of UNC-51-like kinase 1 is a novel biomarker of poor prognosis in patients with esophageal squamous cell carcinoma. Cancer Sci 102: 1568-1575, 2011.

14. Tang J, Deng R, Luo RZ, Shen GP, Cai MY, Du ZM, Jiang S, Yang MT, Fu JH and Zhu XF: Low expression of ULK1 is associated with operable breast cancer progression and is an adverse prognostic marker of survival for patients. Breast Cancer Res Treat 134: 549-560, 2012.

15. Cai MY, Hou JH, Rao HL, Luo RZ, Li M, Pei XQ, Lin MC, Guan XY, Kung HF, Zeng YX and Xie D: High expression of H3K 27me3 in human hepatocellular carcinomas correlates closely with vascular invasion and predicts worse prognosis in patients. Mol Med 17: 12-20, 2011.

16. Camp RL, Dolled-Filhart M and Rimm DL: X-tile: A new bio-informatics tool for biomarker assessment and outcome-based cut-point optimization. Clin Cancer Res 10: 7252-7259, 2004.

17. Raeside DE: Monte Carlo principles and applications. Phys Med Biol 21: 181-197, 1976.

18. Brezden-Masley C and Polenz C: Current practices and challenges of adjuvant chemotherapy in patients with colorectal cancer. Surg Oncol Clin N Am 23: 49-58, 2014.

19. O'Connell JB, Maggard MA and Ko CY: Colon cancer survival rates with the new American Joint Committee on Cancer sixth edition staging. J Natl Cancer Inst 96: 1420-1425, 2004.

20. Oh HS, Chung HJ, Kim HK and Choi JS: Differences in overall survival when colorectal cancer patients are stratified into new TNM staging strategy. Cancer Res Treat 39: 61-64, 2007.

21. Kim KH, Yang SS, Yoon YS, Lim SB, Yu CS and Kim JC: Validation of the seventh edition of the American Joint Committee on Cancer tumor-node-metastasis (AJCC TNM) staging in patients with stage II and stage III colorectal carcinoma: Analysis of 2511 cases from a medical centre in Korea. Colorectal Dis 13: e220-e226, 2011

22. Ross JS: Biomarker-based selection of therapy for colorectal cancer. Biomarkers Med 5: 319-332, 2011.

23. Rizzo S, Bronte G, Fanale D, Corsini L, Silvestris N, Santini D, Gulotta G, Bazan V, Gebbia N, Fulfaro F and Russo A: Prognostic vs predictive molecular biomarkers in colorectal cancer: Is KRAS and BRAF wild type status required for anti-EGFR therapy? Cancer Treat Rev 36 (Suppl 3): S56-S61, 2010.

24. Yokota T: Are KRAS/BRAF mutations potent prognostic and/or predictive biomarkers in colorectal cancers? Anticancer Agents Med Chem 12: 163-171, 2012.

25. Hönscheid P, Datta K and Muders MH: Autophagy: Detection, regulation and its role in cancer and therapy response. Int J Radiat Biol 90: 628-635, 2014.

26. Schmukler E, Kloog Y and Pinkas-Kramarski R: Ras and autophagy in cancer development and therapy. Oncotarget 5: 577-586, 2014.

27. Mizushima N: The role of the Atg1/ULK1 complex in autophagy regulation. Curr Opin Cell Biol 22: 132-139, 2010.

28. Mathonnet M, Perraud A, Christou N, Akil H, Melin C, Battu S, Jauberteau MO and Denizot Y: Hallmarks in colorectal cancer: Angiogenesis and cancer stem-like cells. World J Gastroenterol 20: 4189-4196, 2014 